\title{
Attenuation of scopolamine-induced deficits in delayed-matching performance by a new muscarinic agonist
}

\author{
ANGELA C. RUSKE \\ University of Otago, Dunedin, New Zealand \\ A. FISHER \\ Institute for Biological Research, Ness Ziona, Israel \\ and \\ K. GEOFFREY WHITE \\ University of Otago, Dunedin, New Zealand
}

\begin{abstract}
The ability of two novel muscarinic agonists, AF150(S) and AF151(S), to (1) enhance matching accuracy in pigeons in a delayed matching-to-sample procedure and (2) reverse deficits associated with scopolamine administration was investigated. There was no effect of either drug alone on matching accuracy over four dose levels $(0,1,2$ and $4 \mathrm{mg} / \mathrm{kg}$ ) following intraperitoneal (i.p.) injections. Administration of scopolamine $(0.03 \mathrm{mg} / \mathrm{kg})$ i.p. impaired matching accuracy across all retention intervals, suggesting that perceptual or attentional processes were disrupted. AF150(S), but not AF151(S), reversed the deficits associated with scopolamine administration. The present results indicate that the cholinergic system may mediate encoding processes and that AF150(S) may be a possible candidate for alleviating some of the cognitive deficits seen in Alzheimer's disease.
\end{abstract}

Age-related memory loss has been the focus of a great deal of research over the past 40 years. Although the physiological mechanisms involved in the memory process are still not fully understood, it is known that the cholinergic system plays an important role in a variety of cognitive processes, particularly learning and memory (Bartus, Dean, Beer, \& Lippa, 1982). Drachman and Leavitt (1974) found that administration of scopolamine (a centrally acting anticholinergic drug) induced memory deficits in humans that closely resembled those seen in aged subjects. The debilitating effects on memory resulting from cholinergic system disruption have since been found in a variety of species, including pigeons (Savage, Stanchfield, \& Overmier, 1994; Wenger, Hudzik, \& Wright, 1993), rats (Dunnett, 1985), monkeys (Bartus, 1978; Glick \& Jarvik, 1970; Penetar \& McDonough, 1983), and humans (Drachman, 1977; Petersen, 1977). Further, it is believed that the decline of basal forebrain cholinergic neurons in the brains of patients suffering from Alzheimer's disease (AD) may partly underlie the memory deficits associated with the disorder (Coyle, Price, \& DeLong, 1983). Decreased choline acetyltransferase (ChAT) ac-

Some of the present data were reported at the Australasian Winter Conference on Brain Research, Queenstown, New Zealand (August 1995). We thank Neroli Harris and Barry Dingwall and his team for technical assistance, and Emily Cooney, Maureen Howard, and Mike Parkes for assistance during the conducting of the experiment. Correspondence should be addressed to A. C. Ruske, Department of Psychology, University of Otago, Dunedin, New Zealand (e-mail: aruske@psy.otago.ac.nz). tivity in the cerebral cortex and hippocampus of AD patients has been commonly reported (Bartus et al., 1982; Volger, 1991) and has been reliably associated with the severity of cognitive deficits in AD (Perry et al., 1978; Volger, 1991).

Of the cholinergic receptors, it is believed that the M1 muscarinic receptor subtype may play an important role in learning and memory (Mash, Flynn, \& Potter, 1985; Whitehouse, 1986). The limited clinical utility of cholinergic agents may be due to the fact that most muscarinic agonists studied to date are selective for the M2 subtype (Davis et al., 1993). As a result, there has been a great deal of interest in developing agents that act selectively on the M1 receptor. Fisher et al. (1993) reported the development of two new Ml agonists, AF150(S) and AF151(S). The new, functionally selective partial Ml agonist, AF150(S)[1-methyl-piperidine-4-spiro-(2'-methylthiazoline $)$, is highly selective toward the $\mathrm{Ml}$ receptor, has a wide therapeutic window, and readily crosses the blood-brain barrier (Brandeis et al., 1995). Both are highly selective full agonists in CHO cells stably transfected with cloned $\mathrm{ml}$ muscarinic receptors, and AF $150(\mathrm{~S})$ is a partial agonist in stimulating phosphoinositides hydrolysis in $\mathrm{CHO}$ cells (Fisher et al., 1993). Brandeis et al. investigated the pharmacodynamic profile of AF150(S) and its ability to reverse AF64A-induced cognitive deficits in rats. They found the lethal dose of AF150(S) to be greater than $500 \mathrm{mg} / \mathrm{kg}$ and the sign-free dose to be greater than $40 \mathrm{mg} / \mathrm{kg}$, indicating a wide therapeutic window. Following bilateral intraventricular administration of AF $64 \mathrm{~A}$ 
Table 1

Sequence of Experimental Conditions for Scopolamine, AF150(S), and AF151(S) Administration

\begin{tabular}{ll}
\hline \multicolumn{1}{c}{ Condition } & \multicolumn{1}{c}{ Session Number } \\
\hline 1. Baseline & $1-72,75,78-79,82,85-86,89,92-93,96$, \\
& $99-100,103,106-107,110,113-114$, \\
& $117,120-166,168-170,172-173,175-177$, \\
& $179-180,182-184,186-187,189-191$, \\
& $193-194,196-198,200-201,203-205$, \\
& $207-208,210-212,214-215,217-219$, \\
& $221-257,259-260,262-264,266-267$, \\
2. $1 \mathrm{mg} / \mathrm{kg} \mathrm{AF} 150(\mathrm{~S})$ & $73-74,90-91$ \\
3. $2 \mathrm{mg} / \mathrm{kg} \mathrm{AF} 150(\mathrm{~S})$ & $76-77,94-95$ \\
4. $4 \mathrm{mg} / \mathrm{kg} \mathrm{AF150(S)}$ & $80-81,87-88$ \\
5. Double distilled water & $83-84,97-98$ \\
6. $1 \mathrm{mg} / \mathrm{kg} \mathrm{AF151(S)}$ & $101-102,115-116$ \\
7.2 mg/kg AF151(S) & $108-109,118-119$ \\
8. $4 \mathrm{mg} / \mathrm{kg} \mathrm{AF151(S)}$ & $104-105,111-112$ \\
9. $0.03 \mathrm{mg} / \mathrm{kg} \mathrm{scopolamine}$ & $167,171,174,178$ \\
10. Saline & $181,185,188,192$ \\
11. Scp + DDW (AF150(S)) & 195,220 \\
12. Scp + 1 mg/kg AF150(S) & 199,216 \\
13. Scp + 2 mg/kg AF150(S) & 202,213 \\
14. Scp + 4 mg/kg AF150(S) & 206,209 \\
15. Scp + DDW (AF151(S)) & 258,282 \\
16. Scp + 1 mg/kg AF151(S) & 261,279 \\
17. Scp + 2 mg/kg AF151(S) & 265,275 \\
18. Scp + 4 mg/kg AF151(S) & 268,272 \\
\hline Not Scp 0.03 mg/kg of scopolat &
\end{tabular}

Note-Scp, $0.03 \mathrm{mg} / \mathrm{kg}$ of scopolamine.

(a neurotoxin that selectively destroys cholinergic neurons in the basal forebrain), rats were tested on a variety of behavioral tasks with doses of 0 (double distilled water [DDW]), $0.5,1$, and $5 \mathrm{mg} / \mathrm{kg}$ of AF150(S). Brandeis et al. found a significant attenuation of $\mathrm{AF} 64 \mathrm{~A}$-induced impairments in all tasks. The most consistent dose at attenuating impairments across tasks was the $1-\mathrm{mg} / \mathrm{kg}$ dose level.

The aim of the present experiment was to test the ability of the muscarinic agonists AF150(S) and AF151(S) to enhance accuracy in the delayed matching-to-sample (DMTS) procedure and to test whether either compound is capable of reversing scopolamine-induced impairments in performance. DMTS is a popular procedure for studying the effects of drugs on memory performance in animals (White, Ruske, \& Colombo, 1996). In the standard procedure, animals are presented with a sample stimulus that they are required to remember over some retention interval. Following the completion of the retention interval, the animal is presented with two choice stimuli, one of which is the same as the sample. Reward is given if the animal chooses the stimulus that matches the sample. The DMTS procedure has been assumed to be sensitive to short-term memory processes and has many similarities to the classic procedure devised by Peterson and Peterson (1959) for studying short-term memory in humans. Scopolamine was employed to generate a performance deficit for two reasons. First, scopolamine is a cholinergic antagonist, and its effects can therefore be understood in terms of the cholinergic hypothesis. Second, although there is no animal model that completely reinstates the pathological and cognitive deficits of $\mathrm{AD}$, scopolamine has been shown to be useful at mimicking at least some of the cognitive deficits present in Alzheimer's patients (Ebert, Ramezani, Wesnes, \& Kirch, 1997).

\section{METHOD}

\section{Subjects}

Four adult homing pigeons with previous histories in DMTS procedures, but no history of drug administration, were maintained at $85 \%$ of their free-feeding weight by supplementary feeding of mixed grain. Water and grit were available at all times in their home cages. Each bird was run 7 days a week unless its weight was $10 \mathrm{~g}$ above or below the prescribed weight.

\section{Apparatus}

A light- and sound-attenuating experimental chamber $(33 \times 33 \times$ $34 \mathrm{~cm}$ ) contained an interface panel on one wall. The interior of the chamber was painted matt black, and a ventilation fan was situated in the right rear of the chamber opposite the interface panel. A Plexiglas response key, $2.2 \mathrm{~cm}$ in diameter, was mounted $22 \mathrm{~cm}$ above the grid floor in the center of the panel. Two side keys, both $2.8 \mathrm{~cm}$ in diameter, were located $10 \mathrm{~cm}$ on either side of the center key, $22 \mathrm{~cm}$ above the grid floor. A force of approximately $0.15 \mathrm{~N}$ was required to operate the microswitch behind the center key and two side keys. In-line displays were mounted behind each key. Each of the two side keys could be illuminated green or red. A vertical line or a dot could be displayed on the center key. Access to wheat was provided via a central hopper located directly beneath the center key. Availability of wheat was signaled by a light located at the hopper opening. Ex- 


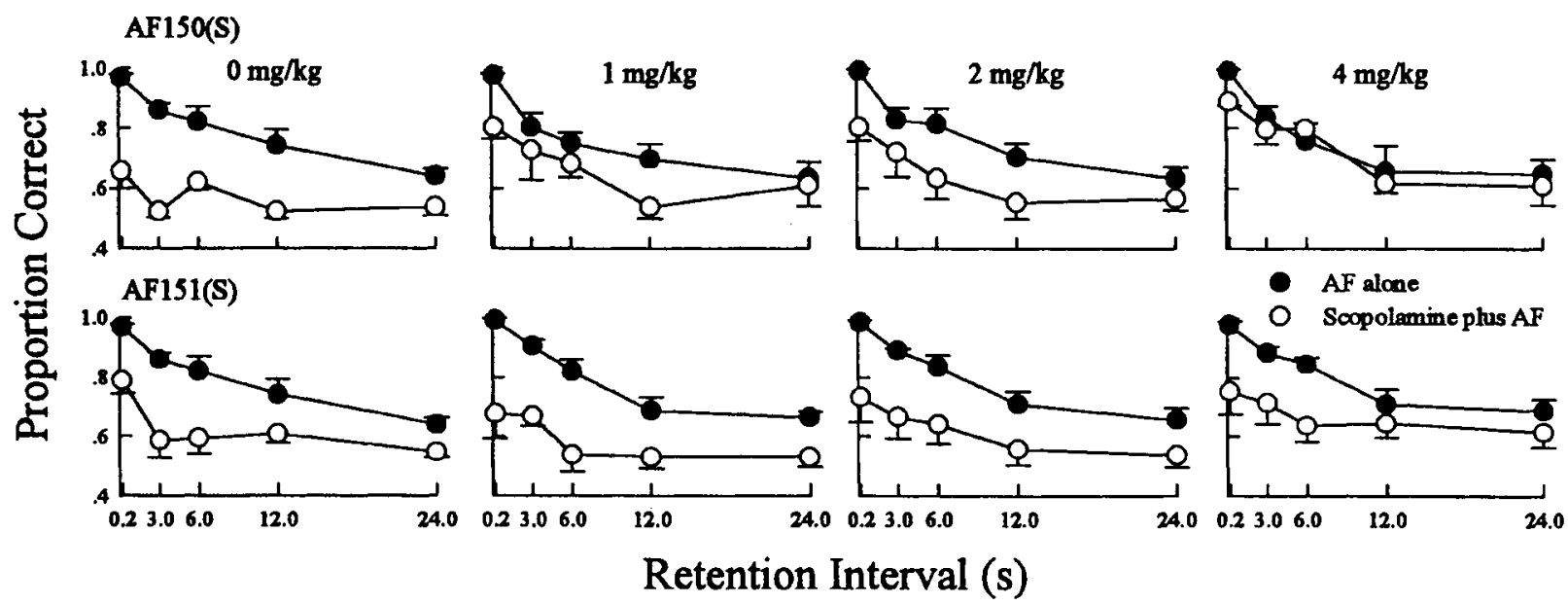

Figure 1. Proportion correct responses as a function of retention interval for each dose level of AF150(S) (top row) and AF151(S) (bottom row). Filled circles represent the administration of AF 150(S) alone and AF151(S) alone, and unfilled circles represent performance following the administration of scopolamine-plus-AF150(S) and scopolamine-plus-AF151(S). Vertical lines refer to SEMs.

perimental events were programmed and responses recorded by a 486 microcomputer with Med-PC software and interfacing located in an adjacent room.

\section{Behavioral Procedure}

Daily experimental sessions consisted of 81 trials or $50 \mathrm{~min}$, whichever came first. The first trial at the beginning of each session did not contribute to data analysis. Each trial began with illumination of the center key with either a vertical line or dot. After five responses on the center key, the chamber was darkened and a retention interval of $0.2,3,6,12$, or $24 \mathrm{sec}$ was initiated. All five retention intervals were scheduled to occur equally often and in a random order within each session. All responses during the retention interval were ineffective. Following the retention interval, the two side keys were illuminated red and green. A correct matching response occurred if the bird pecked red when the sample stimulus was a dot and if the bird pecked green when the sample was a vertical line (technically, "symbolic" DMTS). Correct responses were reinforced with 2.5 -sec access to wheat. Incorrect responses resulted in a $2.5-\mathrm{sec}$ blackout period. Trials were separated by a 5 -sec intertrial interval. The red and green choice stimuli were presented equally often on left and right keys for each retention interval. Preliminary baseline training in this procedure continued for 72 sessions before the first drug administration sessions were arranged (Table 1).

\section{Drug Administration}

AF150(S) and AF151(S). The drugs were synthesized and supplied by A.F., who has described their pharmacological properties elsewhere (Fisher et al., 1993). Drug administration sessions were conducted on Mondays, Tuesdays, Thursdays, and Fridays, with continued baseline training on every other day over a period of 28 days (Table 1). AF 150(S) and AF151(S) were diluted to their required concentrations with DDW. The four drug concentrations used were 1,2 , and $4 \mathrm{mg} / \mathrm{ml}$, plus vehicle $(0 \mathrm{mg} / \mathrm{ml})$. Drugs were administered via the intraperitoneal (i.p.) cavity at a constant volume of $1 \mathrm{ml} / \mathrm{kg} 20 \mathrm{~min}$ prior to behavioral testing. The order of drug administration was the same for each bird. Each of the three dose levels, as well as the vehicle control, was administered in random order, except that each dose was tested on 2 consecutive days (Table 1). Seven sessions of baseline training separated AF150(S) testing and testing with AF $151(\mathrm{~S})$. Birds received four sessions under each dose level of drug plus vehicle.
Scopolamine plus AF150(S) and AF151(S). The concentration of $0.03 \mathrm{mg} / \mathrm{ml}$ of scopolamine hydrobromide was chosen on the basis of previous research (Savage et al., 1994; Teal \& Evans, 1982) and our own pilot studies. Combined administration of $0.03 \mathrm{mg} / \mathrm{ml}$ of scopolamine with each of the four dose levels of AF150(S) and AF $151(S)(0,1,2$, and $4 \mathrm{mg} / \mathrm{kg})$ occurred on Tuesdays and Fridays of each week. Birds were maintained on baseline training on nondrug days (Table 1). Scopolamine was administered via the i.p. cavity at a constant volume of $1 \mathrm{mg} / \mathrm{ml} 30 \mathrm{~min}$ prior to experimental testing, and AF150(S) or AF151(S) was administered 5 min after the administration of scopolamine. Testing with AF150(S) was completed before testing with AF151(S) began. For both drugs, order of administration for all birds occurred in an ascending then descending series (Table 1). Each bird was tested for two sessions with combined dose levels of scopolamine-plus-AF $150(\mathrm{~S})$ and scopolamineplus-AF151(S).

\section{Data Analysis}

Total correct and error matching responses were collapsed over trial type (dot vs. line samples) and summed over the two sessions of drug or vehicle administration for each retention interval (individual analysis) for the different conditions of AF 150(S), AF151(S), and scopolamine administration. Proportions of correct responses were calculated by dividing the total number of correct matching responses by the total number of responses (correct plus error) at each retention interval.

Because the proportion correct measure is susceptible to response bias and is bounded at 1.0, we also report estimates of discriminability derived from the choice theory of signal detection theory (White et al., 1996). The discriminability measure does not have an upper bound and is therefore a more sensitive measure at high levels of accuracy. Discriminability measures were calculated for individual birds and averaged across birds to obtain group means at each retention interval. In order to account for zeros appearing in some cells, which results in indeterminate measures, 0.5 was added to each total of every cell (Hautus, 1995). Estimates of discriminability at each retention interval $(\log d)$ were derived from correct $(c)$ and error $(e)$ responses following each sample $(1,2)$ according to Equation 1 (White, 1985).

$$
\log d=0.5 \times \log (c 1 / e 1 \times c 2 / e 2)
$$

Higher order measures of performance were achieved by fitting a negative exponential function to the individual and group mean 


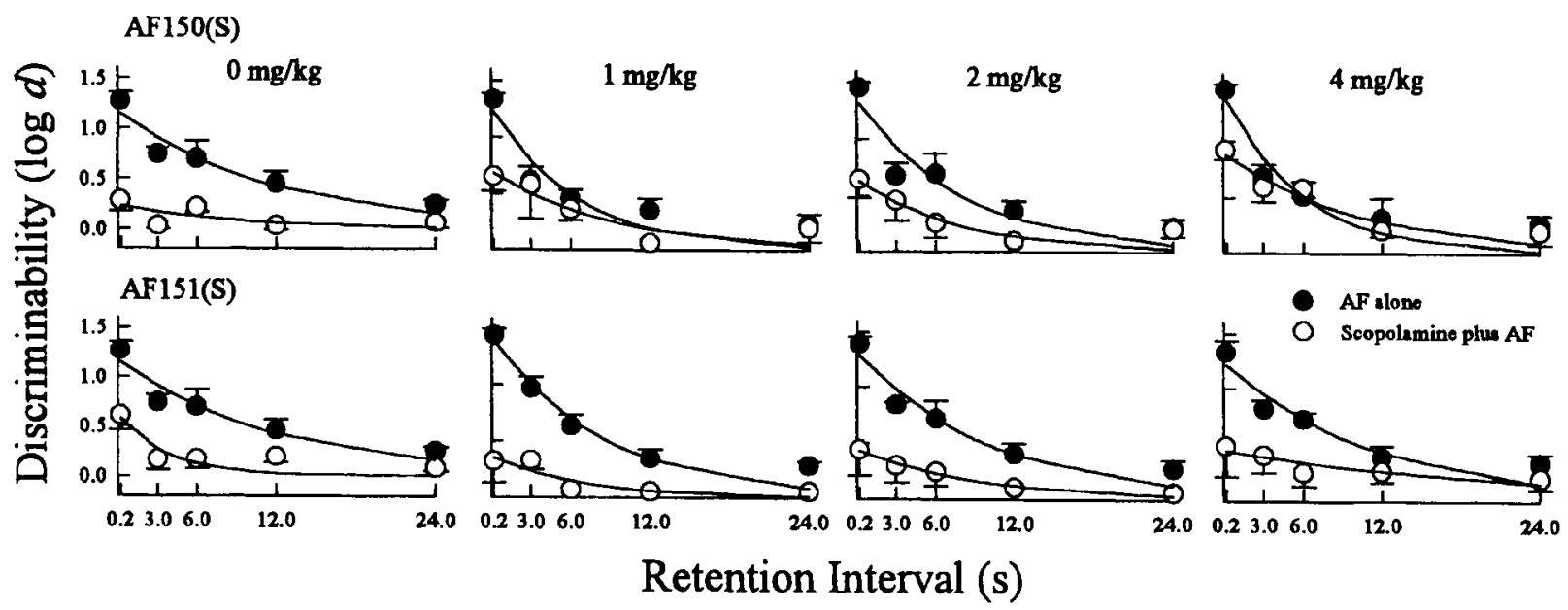

Figure 2. Discriminability measures $(\log d)$ as a function of retention interval for each dose level of $\mathrm{AF150(S)}$ (top row) and AF151(S) (bottom row). Filled circles represent the administration of AF150(S) alone and AF151(S) alone, and unfilled circles represent performance following the administration of scopolamine-plus-AF150(S) and scopolamine-plus-AF151(S). Vertical lines refer to $S E M$ s.

discriminability measures $y$ for the different retention intervals $t$ (White, 1985; White \& Harper, 1996). This function is given by Equation 2.

$$
y=a \times \exp [-b \times \operatorname{sqrt}(t)] .
$$

The advantage of fitting such a function to the data is that it allows an assessment of the rate of forgetting independently of overall level of performance or initial discriminability (discriminability at time $t=$ 0 ). These independent aspects of remembering are estimated by the parameters $b$ and $a$, respectively, of the fitted functions (White, 1985). The utility of this higher order analysis is that it separates encoding or attentional aspects of performance, measured by initial discriminability, from retrieval or memorial aspects, measured by rate of forgetting. These different aspects of DMTS performance are often sensitive to the effects of different drugs (White et al., 1996). For example, the attentional component, as measured by the initial discriminability parameter $a$, is influenced by scopolamine (Kirk, White, \& McNaughton, 1988), whereas rate of forgetting, as measured by the parameter $b$, is influenced by chlorpromazine and phenobarbital (Watson \& Blampied, 1989; White, Harper, \& Watson, 1994).

Proportion correct, $\log \left(d^{\prime}\right)$, initial discriminability, and rate of forgetting parameters for each group were subjected to a repeated measures analysis of variance. A criterion of $p<.05$ was required for significance.

\section{RESULTS}

Figure 1 shows mean proportion correct responses as a function of retention interval for each dose level of AF150(S) (top panels) and AF151(S) (bottom panels). The top panels of Figure 1 show performance during the administration of AF $150(\mathrm{~S})$ alone and in combination with scopolamine administration. There was no effect of AF150(S) on matching accuracy for any dose level of drug when administered alone. There was a significant effect of retention interval on performance $[F(4,12)=$ 19.36], as seen by the systematic decrease in matching accuracy with increasing retention interval duration. The debilitating effect of scopolamine administration on matching accuracy can be seen at the $0-\mathrm{mg} / \mathrm{kg}$ condition, in which performance following scopolamine administration was significantly worse than performance during vehicle administration $[F(1,3)=58.05]$.

When AF 150(S) was administered in combination with scopolamine, matching accuracy increased with increasing dose levels of $\mathrm{AF} 150(\mathrm{~S})[F(3,9)=4.56]$. In order to determine which dose levels of AF150(S) significantly improved matching accuracy above the scopolamineplus-vehicle condition, comparisons were made for individual dose levels. There was no significant improvement in matching accuracy at the $1-\mathrm{mg} / \mathrm{kg}$ dose level. At the $2-\mathrm{mg} / \mathrm{kg}$ dose level, there was a significant interaction between dose level and retention interval $[F(4,12)=3.86]$, consistent with an improvement in matching accuracy at the shorter retention intervals. At the 4-mg/ $\mathrm{kg}$ dose level, there was a significant effect of drug on matching accuracy $[F(1,3)=370.09]$, as well as a significant effect of retention interval duration $[F(4,12)=16.45]$. That is, administering $4 \mathrm{mg} / \mathrm{kg}$ of $\mathrm{AF} 150(\mathrm{~S})$ with scopolamine resulted in both an overall improvement in performance and an even greater improvement at short delays compared with performance when scopolamine was administered with vehicle. A comparison between the conditions with $4 \mathrm{mg} / \mathrm{kg}$ of AF150(S) alone and the scopolamine-plus$4 \mathrm{mg} / \mathrm{kg}$ of AF 150 (S) revealed no significant difference in performance as a function of drug condition, suggesting that $4 \mathrm{mg} / \mathrm{kg}$ of AF 150 (S) completely ameliorated the effects of scopolamine.

The bottom panels of Figure 1 show the performance during the administration of AF151(S) alone and in combination with scopolamine administration. Administration of AF151(s) alone produced no significant improvement in matching accuracy. There was a significant effect of retention interval on performance $[F(4,12)=26.29]$, as shown by the systematic decrease in matching accuracy as retention interval duration increased. As with earlier testing with AF150(S), scopolamine significantly 


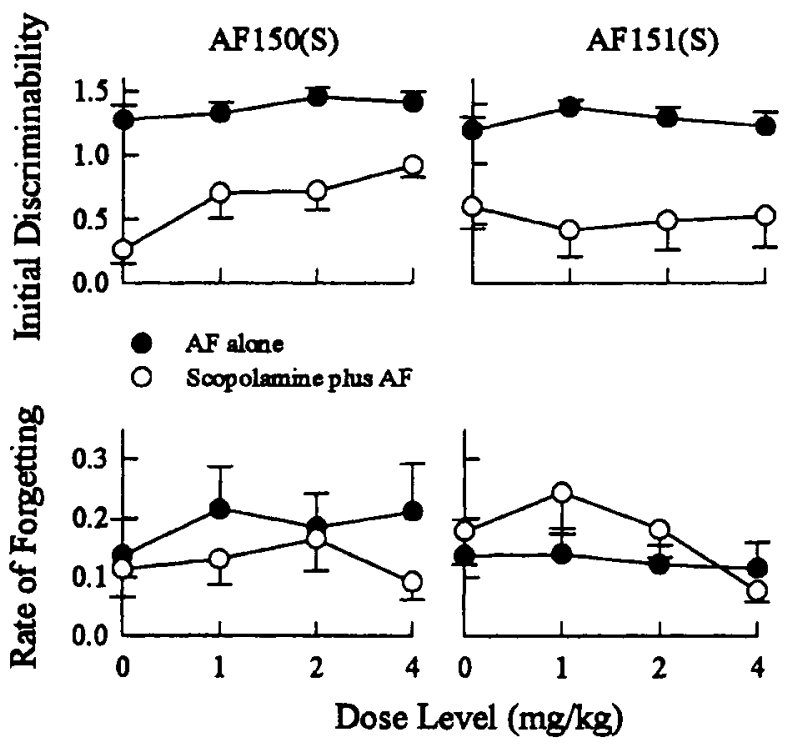

Figure 3. Initial discriminability (top panels) and rate-offorgetting (bottom panels) parameters as a function of dose level for AF150(s) (left two panels) and AF151(S) (right two panels). Filled circles represent the administration of $A F 150(S)$ alone and AF151(S) alone, and unfilled circles represent performance following the administration of scopolamine-plus-AF150(S) and scopolamine-plus-AF151(S). Vertical lines refer to $S E M$ s.

reduced matching accuracy compared with the vehicle control condition $[F(1,3)=14.71]$, although this disruption was not as marked as in the earlier testing with AF 150(S). No dose level of AF151(S) was capable of reversing any of the deficits associated with scopolamine administration.

Figure 2 shows mean discriminability measures $(\log d)$ as a function of retention interval for each dose level of AF150(S) (top panels) and AF15I(S) (bottom panels). In the AF150(S)-alone condition, there was no significant improvement in performance above vehicle at any dose level. In the scopolamine-plus-AF150(S) condition, there was a significant improvement in matching accuracy as a function of dose level $[F(3,9)=4.5]$. The improvement in performance was dose dependent, as evidenced by the linear relationship between dose level and performance $[F(1,3)=49.0]$. A more detailed comparison was made between scopolamine combined with vehicle and each of the other three dose levels of AF150(S) in order to determine which dose levels significantly enhanced performance. There was a significant dose $\times$ retention interval interaction at the $2-\mathrm{mg} / \mathrm{kg}[F(4,12)=3.4]$ and $4-\mathrm{mg} / \mathrm{kg}$ levels $[F(4,12)=3.2]$, consistent with enhanced performance at the shorter retention intervals with AF150(S) administration. At the 4- $\mathrm{mg} / \mathrm{kg}$ dose level of AF150(S), there was also a significant increase in overall matching accuracy compared with performance during the scopolamine-plus-vehicle condition $[F(1,3)=278.3]$.

The bottom panels of Figure 2 show performance following the administration of AF151(S) alone plotted with performance following the combined administration of scopolamine-plus-AF151(S). In the AF151(S)-alone condition, performance was similar to that in the AF150(S)alone condition. That is, performance did not change significantly as a function of dose level. There was a significant effect of retention interval on matching accuracy $[F(4,12)=28.8]$, as seen by the systematic decrease in performance as retention interval duration increased. In the combined conditions, there was no noticeable attenuation of scopolamine-induced impairments at any dose level of AF151(S).

The top panels of Figure 3 show the group mean initial discriminability parameters, and the bottom panels of Figure 3 show the group mean rate-of-forgetting parameters, derived from fitting the negative exponential equation to individual log discriminability measures, as a function of dose level for AF150(S) (left two panels) and AF151(S) (right two panels). There was no effect of dose on initial discriminability in the AF150(S)-alone condition, which is consistent with the above-mentioned analyses, which showed no effect of AF150(S) alone on matching accuracy. The disruption in performance following scopolamine administration was due to a significant decrease in initial discriminability $[F(1,3)=25.30]$ and was not associated with an effect on rate of forgetting. The attenuation of the scopolamine-induced deficits in performance following AF150(S) administration was due to a significant increase in initial discriminability $[F(3,9)=7.1]$. There was no evidence of a decrease in rate of forgetting. Figure 3 shows that the most effective dose of AF150(S) in increasing initial discriminability was the $4-\mathrm{mg} / \mathrm{kg}$ dose.

The right two panels of Figure 3 show initial discriminability and rate-of-forgetting parameters as a function of dose level for the AF151(S)-alone and scopolamineplus-AF151(S) conditions. There was no significant effect of dose on initial discriminability or rate of forgetting in the AF151(S)-alone condition. Scopolamine administration significantly impaired initial discriminability $[F(1,3)=9.29]$ and had no effect on rate of forgetting. No dose level of AF151(S) was capable of ameliorating the effects of scopolamine administration.

\section{DISCUSSION}

Administration of AF150(S) and AF151(S) by themselves did not enhance performance above vehicle control. If either compound was capable of enhancing memory, we might have expected increased matching accuracy as retention interval duration increased. At long retention intervals, discriminability was low overall, thus making it possible for either drug to enhance memory by decreasing rate of forgetting. At short retention intervals, discriminability was high overall, and it was therefore unlikely that either drug would enhance initial discriminability. Order of drug administration during testing of $\mathrm{AF} 150(\mathrm{~S})$ and $\mathrm{AF} 151$ (S) alone was designed to counterbalance order effects, and in any case, as there was no 
improvement in performance over the period of testing, it is unlikely that order of administration contributed to the result.

Scopolamine administration resulted in an overall decrease in matching accuracy, and in particular a reduction in initial discriminability as measured by the intercept parameter $a$ of the negative exponential function. The scopolamine-induced reduction was attenuated by administration of $\mathrm{AF} 150(\mathrm{~S})$. Thus, when scopolamine was combined with increasing doses of AF $150(\mathrm{~S})$, initial discriminability $(a)$ systematically increased, and there was no change in the rate of forgetting $(b)$. The ability of $\mathrm{AF} 150(\mathrm{~S})$ to attenuate scopolamine-induced deficits supports the notion that enhanced cholinergic function reduces the severity of amnesic effects associated with cholinergic system dysfunction (Mash et al., 1985; Perry, 1986). The present result suggests that the mode of action may be through attention or other processes related to encoding. The effect of AF150(S) in the present study was similar to that in the study by Brandeis et al. (1995), in which AF 150 (S) partially reversed deficits in water maze and radial arm maze performance produced by AF64A.

AF151(S) did not reverse the deficits associated with scopolamine administration, and at some dose levels, it appeared to slightly enhance the effects of scopolamine. It is possible that the difference in effectiveness between the two agonists may be due to the slightly different pharmacodynamic properties of each compound. For example, $\mathrm{AF} 150(\mathrm{~S})$ is a partial agonist in stimulating phosphoinositide hydrolysis, whereas AF151(S) is a full agonist (Fisher et al., 1993). Also, the ability of AF150(S) to reverse scopolamine-induced deficits may be attributed to its high selectivity toward $\mathrm{m} 1$ receptors (Fisher et al., 1993) and its ability to mediate distinct $m_{1}$ AChR signaling pathways (Brandeis et al., 1995).

The ability of AF150(S) to attenuate a scopolamineinduced deficit further highlights the facilitating effects selective M1 agonists play in reversing deficits associated with cholinergic system disruption. Previously, AF102B, a highly selective agonist to the M1 receptor (Fisher et al., 1991; Ono, Saito, Ohgane, Kawanishi, \& Mizobe, 1988) was shown to be successful at enhancing cognitive ability in aged rats (Brandeis, Dachir, Sapir, Levy, \& Fisher, 1990), AF64A-treated rats (Fisher et al., 1989), and AF64A-treated mice (Fisher et al., 1991). In previous studies with humans, muscarinic agonists, such as oxotremorine, arecoline, and RS86, have shown modest cognitive improvement, but their therapeutic use has been hindered due to the production of adverse side effects, such as hypersalivation, chills, and tremor (Hollander et al., 1987; Hollander, Mohs, \& Davis, 1986). It is becoming increasingly likely that the success of treatments for neurological or age-related memory disorders is dependent not only on their ability to reverse some, or all, of the cognitive deficits associated with the disorders, but also on the degree of their selectivity to the M1 receptor.

The effects of scopolamine on the pigeons' performance in the present study parallel those reported in other species. For example, Kirk et al. (1988) administered scopolamine to rats in a delayed matching-to-position procedure. They found that rats' performance decreased in a dose-related manner and noted a systematic decrease in initial discriminability with increasing dose level, even at the lowest dose $(0.005 \mathrm{mg} / \mathrm{kg})$. There was no change in the rate-of-forgetting parameter as a function of dose level. A reanalysis of data from Bartus (1978) in a review by White et al. (1996) showed that scopolamine decreased initial discriminability, but not rate of forgetting, in rhesus monkeys in a delayed response procedure. Dunnett (1985) administered four dose levels of scopolamine to rats performing a delayed matching-to-position task. The dose-related decrease in matching accuracy was obvious at short retention intervals. Similar results have also been found in studies of humans. Safer and Allen (1971) administered scopolamine to young healthy adults and tested their ability to recall digits after retention intervals of 0,4 , and $20 \mathrm{sec}$. Our reanalysis of their data revealed that scopolamine administration decreased initial discriminability relative to baseline and that rate of forgetting was similar in both conditions.

Further, the deficits produced by scopolamine administration are similar to those produced by other manipulations of the cholinergic systems. For example, Bymaster, Heath, Hendrix, and Shannon (1993) found that scopolamine, trihexyphenidyl, and pirenzepine (all centrally acting muscarinic antagonists) impaired rats' performance in a spatial alternation task in a similar manner. Penetar and McDonough (1983) found that the anticholinergic atropine impaired monkeys' performance in a DMTS task. In particular, the highest dose level $(0.44 \mathrm{mg} / \mathrm{kg})$ resulted in a noticeable decrease in matching accuracy at the 0 -sec retention interval. Dunnett (1985) found that ibotenic acid lesions of the nucleus basalis in rats resulted in impaired performance at all retention intervals.

It appears that the effects of scopolamine mimic some of the amnesic effects found in patients suffering from $\mathrm{AD}$. For example, Money, Kirk, and McNaughton (1992) compared the performance of patients diagnosed with senile dementia of the Alzheimer's type to that of age-matched controls in a DMTS procedure. They found a marked decrease in performance in $\mathrm{AD}$ patients relative to control subjects and suggested that this deficit was due to a decrease in the initial discriminability parameter and not to changes in rate of forgetting. Other studies that have investigated behavioral differences between $A D$ patients and age-matched controls have also shown a marked decrease in matching accuracy at short retention intervals (Kopelman, 1985; Lange, Sahakin, Quinn, Marsden, \& Robbins, 1995; Sahgal et al., 1992).

Disruption to the cholinergic system (and debilitating effects associated with AD) has predominantly been viewed as affecting retrieval processes. It seems highly possible, however, that when encoding processes, as reflected in the level of initial discriminability, are disentangled from retrieval processes, such as rate of forgetting, at least some of the deficits produced by disruption to the cholinergic system may actually be due to impaired encoding ability. 
The majority of animal studies investigating drug effects on performance have been conducted on either rats or monkeys. The use of pigeons in the present study may raise some question as to the generality of the results. It seems, however, that the avian cholinergic system may function in a similar manner to that of other mammals. Medina and Reiner (1994) examined the pigeon brain in order to ascertain the distribution of cholinergic perikarya and fibres. They found that the avian brain possesses major cholinergic inputs, a result consistent with findings from previous studies on avian cholinergic systems (Shimizu \& Karten, 1990; Sorenson \& Chiappinelli, 1992). In particular, they found that the organization of the cholinergic system in the basal forebrain is very similar to that of reptiles and mammals.

Behavioral studies also suggest that the avian cholinergic system plays a role in memory that is similar to that of other species. For example, Teal and Evans (1982) investigated the effects of three doses of scopolamine $(0.01$ to $0.1 \mathrm{mg} / \mathrm{kg}$ ) on matching performance in pigeons. Scopolamine produced a dose-related decrease in performance. A reanalysis (see White et al., 1996) showed that this deficit was manifested as a decrease in initial discriminability, suggesting that the birds had difficulty encoding the stimuli to be remembered. Similar behavioral deficits have also been reported in monkeys (Penetar \& McDonough, 1983), rats (Bartus, 1978), and humans (Safer \& Allen, 1971).

In conclusion, the present finding that $\mathrm{AF} 150$ (S) attenuated a scopolamine-induced deficit in DMTS performance is consistent with the cholinergic hypothesis given that the cholinergic system appears to play a significant role in the encoding of information. Future research could compare the effects of AF150(S) to those of the acetylcholinesterase inhibitor tacrine, which has been reported to alleviate cognitive deficits, but in association with severe peripheral side effects (Beermann, 1993). The ability of AF150(S) to show high selectivity toward Ml muscarinic receptors (therefore minimizing peripheral side effects) and to attenuate performance deficits produced by a cholinergic antagonist has implications for the treatment of $\mathrm{AD}$, in which the cholinergic system may play an important role.

\section{REFERENCES}

BaRTUS, R. T. (1978). Evidence for a direct cholinergic involvement in the scopolamine-induced amnesia in monkeys: Effects of concurrent administration of physostigmine and methylphenidate with scopolamine. Pharmacology, Biochemistry \& Behavior, 9, 833-836.

Bartus, R. T., Dean, R. L., BeEr, B., \& LipPa, A. S. (1982). The cholinergic hypothesis of geriatric memory dysfunction. Science, 217, 408417.

BEERMANN, B. (1993). Side effects of long acting cholinesterase inhibitors. Acta Neurologica Scandinavica, 88 (Suppl. 149), 53-54.

Brandeis, R., Dachir, S., SAPIR, M., LeVy, A., \& Fisher, A. (1990). Reversal of age-related cognitive impairments by an Ml cholinergic agonist, AF 102B. Pharmacology, Biochemistry \& Behavior, 36, 89-95.

Brandeis, R., Sapir, M., Hafif, N., Abraham, S., Oz, N., Stein, E., \& FisHer, A. (1995). AF150(S): A new functionally selective Ml agonist improves cognitive performance in rats. Pharmacology, Biochemistry \& Behavior, 51, 667-674.
Bymaster, F. P., Heath, I., Hendrix, J. C., \& Shannon, H. E. (1993). Comparative behavioral and neurochemical activities of cholinergic antagonists in rats. Journal of Pharmacology \& Experimental Therapeutics, 267, 16-24.

CoYle, J. T., Price, D. L., \& DeLong, M. R. (1983). Alzheimer's disease: A disorder of cortical cholinergic innervation. Science, 219 , 1184-1190.

Davis, R., Raby, C., Callahan, M. J., Lipinski, W., Schwarz, R., Dudley, D. T., Lauffer, D., Reece, P., Jaen, J., \& TeCle, H. (1993). Subtype selective muscarinic agonists: Potential therapeutic agents for Alzheimer's disease. Progress in Brain Research, 98, 439-445.

Drachman, D. A. (1977). Memory and cognitive function in man: Does the cholinergic system have a specific role? Neurology, 27, 783-790.

Drachman, D. A., \& LeavitT, J. L. (1974). Human memory and the cholinergic system. A relationship to aging? Archives of Neurology, 30, 113-121.

DunNETT, S. B. (1985). Comparative effects of cholinergic drugs and lesions of nucleus basalis or fimbria-fornix on delayed matching in rats. Psychopharmacology, 87, 357-363.

Ebert, U., Ramezani, E., Wesnes, K. A., \& Kirch, W. (1997). The dose-dependent, but temporary, ability of physostigmine to counteract the effects of scopolamine on cognitive function in young volunteers. Proceedings of the British Psychology Society, 5, 48.

Fisher, A., Brandeis, R., Karton, I., PitTel, Z., Gurwitz, D., HaRING, R., SAPIR, M., Levy, A., \& Heldman, E. (1991). ( \pm )-cis-2Methyl-spiro(1,3-oxathiolane-5,3')quinuclidine, an Ml selective cholinergic agonist, attenuates cognitive dysfunctions in an animal model of Alzheimer's disease. Journal of Pharmacology \& Experimental Therapeutics, 257, 392-403.

Fisher, A., Brandeis, R., Pittel, Z., Karton, I., Sapir, M., Dachir, S., LeVy, A., \& Heldman, E. (1989). ( \pm )-cis-2-Methyl-spiro(1,3oxathiolane-5, $3^{\prime}$ )quinuclidine (AF102B): A new M1 agonist attenuates cognitive dysfunctions in AF64A-treated rats. Neuroscience Letters, 102, 325-331.

Fisher, A., Heldman, E., Gurwitz, D., Haring, R., Meshulam, H., BrandeIs, R., SaPIR, M., Marcianno, D., Barak, D., Vogel, Z., \& KARTON, Y. (1993). AF150(S) and AF151(S): New Ml agonists mediate $\mathrm{ml}$ selective signalling, neurotrophic-like effects and restore AF64A cognitive deficits in rats. Society for Neuroscience Abstracts, $19,1767$.

GLICK, S. D., \& JARvIK, M. E. (1970). Differential effects of amphetamine and scopolamine upon matching performance of monkeys with lateral frontal lesions. Journal of Comparative Physiological Psychology, 73, 307-313.

HaUtus, M. J. (1995). Corrections for extreme proportions and their biasing effects on estimated values of $d^{\prime}$. Behavioral Research Methods, Instruments, \& Computers, 27, 46-51.

Hollander, E., Davidson, M., Mohs, R. C., Horvath, T. B., Davis, B. M., ZemishlanY, Z., \& Davis, K. L. (1987). RS 86 in the treatment of Alzheimer's disease: Cognitive and biological effects. Biological Psychiatry, 22, 1067-1078.

Hollander, E., Mohs, R. C., \& Davis, K. L. (1986). Cholinergic approaches to the treatment of Alzheimer's disease. British Medical Bulletin, 42, 97-100.

Kirk, R. C., White, K. G., \& McNaughton, N. (1988). Low dose scopolamine affects discriminability but not rate of forgetting in delayed conditional discrimination. Psychopharmacology, 96, 541-546.

Kopelman, M. D. (1985). Rate of forgetting in Alzheimer-type dementia and Korsakoff's syndrome. Neuropsychologia, 23, 623-638.

Lange, K. W., Sahakin, B. J., Quinn, N. P., Marsden, C. D., \& RobBINS, T. W. (1995). Comparison of executive and visuospatial memory function in Huntington's disease and dementia of Alzheimer type matched for degree of dementia. Journal of Neurology, Neurosurgery \& Psychiatry, 58, 598-606.

Mash, D. C., FlynN, D. D., \& PotTer, L. T. (1985). Loss of M2 muscarinic receptors in the cerebral cortex in Alzheimer's disease and experimental cholinergic denervation. Science, 228, 115-117.

MEDINA, L., \& REINER, A. (1994). Distribution of choline acetyltransferase immunoreactivity in the pigeon brain. Journal of Comparative Neurology, 342, 497-537.

MoneY, E. A., KiRK, R. C., \& McNaughton, N. (1992). Alzheimer's dementia produces a loss of discrimination but no increase in rate of 
memory decay in delayed matching to sample. Neuropsychologia, $\mathbf{3 0}$ 133-143.

Ono, S., Saito, Y., Ohgane, N., Kawanishi, G., \& Mizobe, F. (1988). Heterogeneity of muscarinic autoreceptors and heteroreceptors in the rat brain: Effects of a novel Ml agonist, AF102B. European Journal of Pharmacology, 155, 77-84.

Penetar, D. M., \& MCDonough, J. H. (1983). Effects of cholinergic drugs on delayed matching-to-sample performance of rhesus monkeys. Pharmacology, Biochemistry \& Behavior, 19, 963-967.

PERRY, E. K. (1986). The cholinergic-hypothesis--Ten years on. British Medical Bulletin, 42, 63-69.

Perry, E. K., Tomlinson, B. E., Blessed, G., Bergmann, K., Gibson, P., \& PERRY, R. H. (1978). Correlation of cholinergic abnormalities with senile plaques and mental test scores in dementia. British Medical Journal, 2, 1457-1459.

PetERSEN, R. C. (1977). Scopolamine induced learning failures in man. Psychopharmacology, 52, 283-289.

Peterson, L. R., \& Peterson, M. (1959). Short-term retention of individual items. Journal of Experimental Psychology, 58, 193-198.

SAFER, D. J., \& ALLEN, R. P. (1971). The central effects of scopolamine in man. Biological Psychiatry, 3, 347-355.

Sahgal, A., Galloway, P. H., McKeith, I. G., Lloyd, S., Cook, J. H., FERrier, I. N., \& EDWARDSON, J. A. (1992). Matching to sample deficits in patients with senile dementias of the Alzheimer and Lewy body types. Archives of Neurology, 49, 1043-1046.

Savage, L. M., Stanchfield, M. A., \& Overmier, J. B. (1994). The effects of scopolamine, diazepam, and lorazepam on working memory in pigeons: An analysis of reinforcement procedures and sample problem type. Pharmacology, Biochemistry \& Behavior, 48, 183-191.

SHIMIZU, T., \& KARTEN, H. J. (1990). Immunohistochemical analysis of the visual wulst of the pigeons (Columba livia). Journal of Comparative Neurology, 300, 346-369.

Sorenson, E. M., \& Chiappineldi, V. A. (1992). Localization of ${ }^{3} \mathrm{H}-$ nicotine, ${ }^{125}$ I-kappa-bungarotoxin, and ${ }^{125}$ I-alpha-bungarotoxin bind- ing to nicotinic site in the chicken forebrain and midbrain. Journal of Comparative Neurology, 323, 1-12.

TEal, J. J., \& Evans, H. L. (1982). Effects of DDAVP, a vasopressin analog, on delayed matching behavior in the pigeon. Pharmacology, Biochemistry \& Behavior, 17, 1123-1127.

VoLGER, B. W. (1991). Alternatives in the treatment of memory loss in patients with Alzheimer's disease. Ciinical Pharmacy, 10, 447-450.

WATSON, J. E., \& Bl.AMPIED, N. M. (1989). Quantification of the effects of chlorpromazine on performance under delayed matching to sample in pigeons. Journal of Experimental Analysis of Behavior, 51, 317-328.

Wenger, G. R., HudziK, T. J., \& Wright, D. W. (1993). Titrating matching-to-sample performance in pigeons: Effects of diazepam, morphine, and cholinergic agents. Pharmacology, Biochemistry \& Behavior, 46, 435-443.

WHITE, K. G. (1985). Characteristics of forgetting functions. Journal of the Experimental Analysis of Behavior, 44, 15-34.

WhITE, K. G., \& HARPER, D. N. (1996). Quantitative reanalysis of lesion effects on rate of forgetting in macaques. Behavioural Brain Research, 74, 223-227.

White, K. G., Harper, D. N., \& Watson, J. E. (1994). Effects of chronic phenobarbital administration on forgetting functions in pigeons. Pharmacology, Biochemistry \& Behavior, 49, 427-431.

White, K. G., RuSke, A. C., \& Colombo, M. (1996). Memory procedures, performance, and processes in pigeons. Cognitive Brain Research, 3, 309-317.

WhITEHOUSE, P. J. (1986). Neuronal loss and neurotransmitter receptor alterations in Alzheimer's disease. In A. Fisher, I. Hanin, \& C. Lachman (Eds.), Alzheimer's and Parkinson's disease: Strategies for research and development (pp. 185-94). New York: Plenum.

(Manuscript received November 12, 1996; revision accepted for publication March 27, 1997.) 